\title{
Numerical and Experimental Investigation of the Morphology Development of Expansion Clouds by a Powder Jet Flow
}

\author{
H. Tang *, V.A.O. Anjorin, A.J. Morgan, L.C. Wrobel, I.E. Barton, Z. Fan \\ Department of Mechanical Engineering, Brunel University, Uxbridge, Middlesex, UB8 3PH, UK
}

\begin{abstract}
Explosion suppression is often the preferred method of explosion attenuation in industry. The morphology development of suppression clouds is important for the design of necessary coverage of the product. This paper presents a numerical and experimental investigation of the growth of powder dispersion as it expands from a discharge nozzle. A Lagrangian stochastic particle-tracking approach and the RNG $k$ $\varepsilon$ turbulence model are adopted in the flow field solver for the dispersed and continuous phases. The flow fields coupled with the particle interactions are predicted. The dispersion characteristics of the expansion of the powder cloud through a pipe for short intervals of time are investigated. This was compared with (1) captured images from experiments, (2) experimental data, and (3) results of previous simulations. Particle positions along the jet are presented. The effects of flow rate on the development of the cloud and a comparison with experimental results are also presented. It is noted that the coverage of the powder cloud can be controlled by the flow rate of the jet, and the developing length of the cloud is more influenced by the flow rate of jet flow than the developing width. The good qualitative agreements achieved are useful for further optimisation of product design.
\end{abstract}

Key words: jet flow, powder flow, discrete phase flow, turbulent flow 


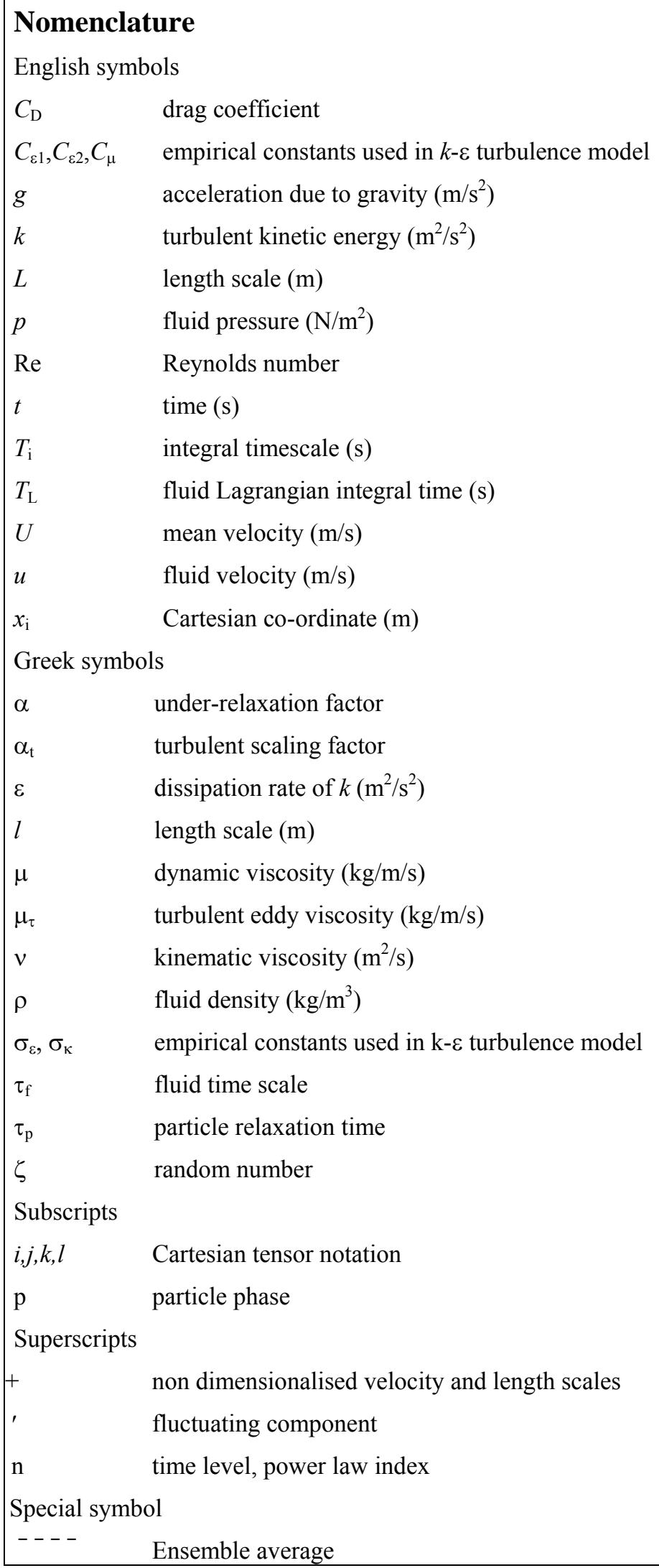




\section{Introduction}

Explosion suppression is often the preferred method of explosion attenuation in industry [1]. Suppression devices invariably use a pressure sensor to detect the explosion at its initial stage of development before releasing a quantity of pressurised powder through a dispersion nozzle in order to extinguish the flame. Eckhoff [2] gives a detailed description of the suppression process. To date, most of the research carried out in this field has focused on experimental work [3]. However, the time consuming and expensive nature of experiments carried out in this area mean that computational studies of the processes taking place will become more important. Areas of chemical plant that need to be protected have invariably different shapes and sizes and it is therefore necessary to know the dispersion characteristics of different types of nozzles. Since the jet progresses at a very high speed, it is currently not possible to experimentally examine anything other than the visual appearance of the jet as it progresses. This is often enough information for the experienced engineer to predict the likely performance of the suppression device. It is generally the case that for a particular type of nozzle to be effective, it needs to fill as much of the volume to be protected as quickly as possible. Preliminary trials [3] have shown that selection based purely on the basis of the pattern of dispersion is an effective way of choosing a nozzle for a given application.

Industrial gas and dust explosions continue to provide a severe threat to the chemical and process industries world-wide. Explosion suppression devices are often employed where ignition prevention measures do not provide the required degree of safety and where the venting of combustion products provides a safety hazard or an environmental risk. In order to continually improve the method by which explosion 
suppression requirements for a given area of plant are calculated, it is necessary to model the discharge characteristics of a suppression device.

Although it is possible to capture the dispersion pattern from a nozzle using highspeed filming techniques, that is an expensive and time consuming procedure, especially if a new nozzle has to be designed and manufactured. It is with this in mind that the authors sought to set-up a simple experimental test case for the dispersion of powder from a pipe-type nozzle and then model the flow and particle dispersion using numerical modelling techniques. It was envisaged that, if this could be carried out successfully, it would subsequently prove possible to design more complicated nozzles on a case by case basis. Experimental data was obtained using a high-speed digital image capture system and dispersion patterns were compared to those obtained using numerical investigations.

In this paper, we present a brief discussion of the Eulerian-Lagrangian model which we have implemented. The model is discussed in three parts; the basic concept of the particle tracking model; the turbulence model and how it is incorporated into the particle model and, finally, how the motion of the particles is coupled with the fluid flow. The paper then presents the experimental results which are compared to the numerical simulation.

Although the present study is concerned with explosion suppression, it is useful to mention other methods available to prevent industrial explosions. These methods include, ignition prevention, inerting and pressure relief venting.

An explosion, being a deflagrative event, takes a finite time to produce a damaging overpressure. This time can be used to both detect and suppress the explosion. Most modern automatic explosion suppression systems are based on a pressure rise detection system which automatically discharges the suppressant rapidly into the 
developing fireball in order to extinguish the flames. An explosion suppression device will typically consist of:

- An extinguishing agent permanently pressurised in an inerting enclosure

- A nozzle designed to disperse the agent

- A fast opening valve for immediate release of suppressant by means of an explosive charge.

A more comprehensive review of dust explosion suppression is presented by Hurlimann [4]. The fundamental behaviour of the flow inside explosion suppressants can be considered as particle-laden flows if materials are powders, which can be investigated numerically and experimentally by various approaches.

\section{Experimental approaches}

2.1 Experimental techniques

Experimental studies of powder jet flow have been reported widely in the last decades. Currently, laser Doppler techniques are used for capturing velocity, particle distribution and size accurately such as laser-Doppler velocimeter (LDV), laser phase Doppler particle anemometer (PDPA), or Particle Image Velocimetry (PIV) [5-7].

\subsection{Experimental set-up}

The objective of the research described here is to visualise the pattern of powder dispersion from a nozzle at short intervals of time. Although this is very much a qualitative approach, knowing the dispersion pattern of a nozzle with time is very useful to the engineer designing suppression systems.

The experimental rig consisted of a standard High Rate Discharge (HRD) suppressor mounted on a steel frame discharging through a $90^{\circ}$ elbow. In order to 
capture images of the dispersion, a Kodak MotionCorder Analyser SR-500 was set up at a right angle to the rig. In order to obtain an idea of the distance travelled by the suppressant, a rope was tied across the area into which the suppressant was to be released and marked at $0.5 \mathrm{~m}$ intervals. Discharges were filmed using a $12 \mathrm{~mm}$ lens at 250 frames per second with a shutter speed of $1 / 3000$ second. Instrumentation employed to measure the pressure decay inside the cylinders consisted of Kistler piezoresistive pressure transducers of type A20 sampling at $1 \mathrm{kHz}$, and a National Instruments data acquisition system.

\section{Numerical approaches}

Consideration of the processes involved in the automatic suppression of dust explosions by inert powders led to the realisation that it is necessary to study complex two-phase flows in some detail. Research into these types of flows is considered important in many engineering disciplines and much experimental and theoretical work has been done in order to further their understanding.

\subsection{Discrete phase method- Lagrangian approach}

In order to track individual droplet behaviour, discrete phase model (DPM) Lagrangian methods will be used with the stochastic Monte-Carlo tracking approach [8] and particle cluster tracking, both of which use statistical methods to trace the turbulent dispersion of particles about the trajectory.

The Lagrangian scheme treats the particle phase as a discrete phase, and the fluid phase as a carrier phase in a Eulerian frame. Trajectories of individual particles can be tracked by balancing the forces acting on them. The Lagrangian scheme is most popular in engineering applications for the prediction of turbulent particulate flows 
because it can easily be combined with a stochastic Monte-Carlo scheme, albeit with high computational cost.

The Lagrangian model has been widely used to predict a variety of two-phase flows since it was first proposed by Migdal and Agosta [8]. The current status of modelling two-phase flows has been reviewed by Sirignano [9], Elghobashi [10], Crowe et al [11], among others.

The Lagrangian model solves Eulerian equations for the fluid phase and then integrates Lagrangian equations of motion for the dispersed phase, tracking individual particles through the flow field. A one-way coupled model assumes that particle motion is influenced by the continuous fluid phase, but the fluid phase is unaffected by the presence of the particles. A two-way coupled model accounts for the two-way exchange of momentum (and energy if applicable) between the dispersed and continuous phases. Source and sink terms are included in the fluid conservation equations and contributions from the particle field are determined.

In recent years, applications of the Lagrangian approach include using the Reynolds stress model (RSM) [12] and direct numerical simulation (DNS) [13-14]. An improved Lagrangian stochastic model with RSM is used by Chen [15] to investigate heavy particle dispersion in turbulent flows. Hagiwara et al[13], Singh et al[16], Sato et al. [14] present the particle behaviour concerning the phase interaction, or turbulence, or heat transfer. Lagrangian methods are still the main approach for particle tracking calculations [17-20]. Liu et al. [21] use a second order momentum turbulence model with the stochastic Monte-Carlo scheme to solve gas-particle flows.

\subsubsection{Governing equations}

The governing equations can be expressed in the following form:

Continuity equation: 


$$
\frac{\partial \rho}{\partial t}+\frac{\partial}{\partial x_{i}}\left(\rho u_{i}\right)=0
$$

For 2D axisymmetric geometries, the continuity equation is given by

$$
\frac{\partial \rho}{\partial t}+\frac{\partial}{\partial x}(\rho u)+\frac{\partial}{\partial r}(\rho v)+\frac{\rho v}{r}=0
$$

Momentum conservation equations:

$$
\frac{\partial}{\partial t}\left(\rho u_{i}\right)+\frac{\partial}{\partial x_{i}}\left(\rho u_{i} u_{j}\right)=-\frac{\partial p}{\partial x_{i}}+\frac{\partial \tau_{i j}}{\partial x_{j}}+\rho g_{i}+F_{i}
$$

where $\tau_{\mathrm{ij}}$ is the stress tensor, $\rho g_{i}$ and $F_{i}$ are the gravitational body force and external body force.

The stress tensor $\tau_{\mathrm{ij}}$ is given by

$$
\tau_{i j}=\left[\mu\left(\frac{\partial u_{i}}{\partial x_{j}}+\frac{\partial u_{j}}{\partial x_{i}}\right)\right]+\frac{2}{3} \mu \frac{\partial u_{l}}{\partial x_{l}} \delta_{i j}
$$

For 2D axisymmetric geometries, the momentum equations are given by

$$
\begin{aligned}
& \frac{\partial}{\partial t}(\rho u)+\frac{1}{r} \frac{\partial}{\partial x}(r \rho u u)+\frac{1}{r} \frac{\partial}{\partial r}(r \rho v u)=-\frac{\partial p}{\partial x}+\frac{1}{r} \frac{\partial}{\partial x}\left[r \mu\left(2 \frac{\partial u}{\partial x}-\frac{2}{3}(\nabla \cdot \vec{v})\right)\right] \\
+ & \frac{1}{r} \frac{\partial}{\partial r}\left[r \mu\left(\frac{\partial u}{\partial r}+\frac{\partial v}{\partial x}\right)\right]+F_{x}
\end{aligned}
$$

and

$$
\begin{aligned}
& \frac{\partial}{\partial t}(\rho v)+\frac{1}{r} \frac{\partial}{\partial x}(r \rho u v)+\frac{1}{r} \frac{\partial}{\partial r}(r \rho v v)=-\frac{\partial p}{\partial r}+\frac{1}{r} \frac{\partial}{\partial x}\left[r \mu\left(\frac{\partial v}{\partial x}+\frac{\partial u}{\partial r}\right)\right] \\
& +\frac{1}{r} \frac{\partial}{\partial r}\left[r \mu\left(2 \frac{\partial v}{\partial r}-\frac{2}{3}(\nabla \cdot \vec{v})\right)\right]-2 \mu \frac{v}{r^{2}}+\frac{2}{3} \frac{\mu}{r}(\nabla \cdot \vec{v})+F_{r}
\end{aligned}
$$

The two-way coupling between the particulate phase and the continuous phase is accomplished by alternately solving their governing equations, which include an interphase exchange of momentum which appears as a momentum sink in the continuous phase momentum equation: 


$$
F=\sum\left(\frac{3 \mu C_{D} \operatorname{Re}}{4 \rho_{p} D_{p}^{2}}\left(u_{p}+u\right)+F_{\text {other }}\right) \dot{m}_{p} \Delta t
$$

where $\mu$ is the viscosity of the fluid, $\rho_{p}$ is the density of the particle, $D_{p}$ is the diameter of the particle, Re is the relative particle Reynolds number, $u_{p}$ is the velocity of the particle, $u$ is the velocity of the fluid, $C_{D}$ is the drag coefficient, $\dot{m}_{p}$ is the mass flow rate of the particles, $\Delta t$ is the time step, $F_{\text {other }}$ denotes other interacting forces.

These equations are coupled with the RNG $k-\varepsilon$ turbulence model. The momentum exchange term is also under-relaxed during the calculation by:

$$
F_{\text {new }}=F_{\text {old }}+\alpha\left(F_{\text {calculated }}-F_{\text {old }}\right)
$$

\subsubsection{Lagrangian Dispersed Phase Model}

The Lagrangian dispersed phase model [8] is used for the prediction of the trajectory of a particle [22]. This is done by integrating the force balance on the particle. The force balance equates the particle inertia with the force acting on the particle, and can be written as:

$$
\frac{d u_{p}}{d t}=F_{D}\left(u-u_{p}\right)+g_{x}\left(\rho_{p}-\rho\right) / \rho_{p}+F_{x}
$$

where $F_{D}\left(u-u_{P}\right)$ is the drag force per unit particle mass and

$$
\begin{gathered}
F_{D}=\frac{18 \mu}{\rho_{P} D_{P}^{2}} \frac{C_{D} \operatorname{Re}}{24} \\
\operatorname{Re}=\frac{\rho D_{P}\left|u_{P}-u\right|}{\mu}
\end{gathered}
$$

The term $F_{x}$ represents additional forces such as thermophoretic force, Saffman's lift force or virtual mass force. However, provided that the particles meet the condition that $\rho_{p}>\rho$; these terms can be neglected, except for the force caused by the pressure gradient in the fluid when there are high acceleration forces present: 


$$
F_{x}=\left(\frac{\rho}{\rho_{p}}\right) u_{p} \frac{\partial u}{\partial x}
$$

The term $g_{x}$ is the gravitational body force. The drag coefficient, $C_{D}$ is given by:

$$
C_{D}=a_{1}+\frac{a_{2}}{R_{e}}+\frac{a_{3}}{R_{e}^{2}}
$$

where the constants are given by Moris and Alexander [23], and take account of ultraStokesian drag.

The momentum transfer from the continuous phase to the particle phase is computed by examining the change in momentum of a particle as it passes through each control volume.This momentum change is computed by Eq. (7).

\subsection{Turbulence modelling - RNG $k-\varepsilon$ model}

The renormalized group (RNG) $k-\varepsilon$ turbulence model [24] is one of the models applied in this research to simulate the shearing-dominated turbulence flow. The RNG-based $k-\varepsilon$ turbulence model is derived from the application of a rigorous statistical technique (renormalization group method [25]) to the instantaneous NavierStokes equations. An improvement of predictions compared to standard (STD) $k-\varepsilon$ turbulence model is reached for transient flows, strained flows, the effect of swirl on turbulence and so on.

The transport equations for the RNG $k-\varepsilon$ turbulence are as follows:

$$
\rho \frac{D k}{D t}=\frac{\partial}{\partial x_{i}}\left(\alpha_{k} \mu_{e f f} \frac{\partial k}{\partial x_{i}}\right)+G_{k}+G_{b}-\rho \varepsilon
$$

and

$$
\rho \frac{D \varepsilon}{D t}=\frac{\partial}{\partial x_{i}}\left(\alpha_{\varepsilon} \mu_{e f f} \frac{\partial \varepsilon}{\partial x_{i}}\right)+C_{1 \varepsilon} \frac{\varepsilon}{k}\left(G_{k}+C_{3 \varepsilon} G_{b}\right)-C_{2 \varepsilon} \rho \frac{\varepsilon^{2}}{k}-R
$$


where $G_{k}=-\rho \overline{\mu_{i}^{\prime} \mu_{j}^{\prime}} \frac{\partial u_{j}}{\partial x_{i}}$, is the generation of turbulent kinetic energy due to the mean velocity gradients, $G_{b}=\beta g_{i} \frac{\mu_{t}}{\operatorname{Pr}_{t}} \frac{\partial T}{\partial x_{i}}$ is the generation of turbulent kinetic energy due to buoyancy, $C_{1 \varepsilon}, C_{2 \varepsilon}$, and $C_{3 \varepsilon}$ are constants, $\alpha_{k}$, and $\alpha_{\varepsilon}$ are the inverse effective Prandtl numbers for $\mathrm{k}$ and $\varepsilon$. The $R$ term in the $\varepsilon$ equation is given by

$$
R=\frac{C_{\mu} \rho \eta^{3}\left(1-\eta / \eta_{0}\right)}{1+\beta \eta^{3}} \frac{\varepsilon^{2}}{k}
$$

where $\eta=S k / \varepsilon, \eta_{0}=4.38, \beta=0.012$.

\subsection{Particle phase tracking - Stochastic Monte-Carlo technique}

Coupling between the particle and continuous phases is achieved by calculating the trajectories of the particles and their impact on the continuous phase, as well as the effect that the particle phase has on the continuum. The two processes of particle and gas interaction are incorporated into the turbulent flow. The solution is accomplished by alternately solving the discrete and continuous phase equations until both phases have converged.

The momentum transfer from the continuous phase to the particle phase is computed by examining the change in momentum of a particle as it passes through each control volume, this momentum change is computed by equation (3).

A stochastic tracking technique $[26,27]$ is used to take into account the effect of the turbulent velocity fluctuations on the particle trajectories. In equation (10), the velocity term includes the instantaneous value of the fluctuating gas flow velocity:

$$
u=\bar{u}+u^{\prime}
$$

in order to predict the dispersion of the particle due to turbulence. By computing the trajectory in this manner for a sufficient number of representative particles, the 
random effects of turbulence on the particles can be modelled. By means of the discrete random walk model $[28,29]$, the fluctuating velocity components apply the discrete piecewise constant functions with respect to time. Also, the random value is kept constant over an interval of time given by the characteristic lifetime of the eddies.

Prediction of particle dispersion makes use of the concept of integral time scale, $T$, which describes the time spent in the turbulence motion along the particle path $d s$ :

$$
T=\int_{0}^{\infty} \frac{u_{p}^{\prime}(t) u_{p}^{\prime}(t+s)}{\overline{u_{p}^{\prime 2}}} d s
$$

$T$ is proportional to the particle dispersion rate, and it can be shown that the particle diffusivity is given by $\overline{u_{i}^{\prime} u_{j}^{\prime}} T$. When particles move with the fluid, the integral time scale becomes the fluid Lagrangian integral time scale $T_{L}$, which can be approximated as:

$$
T_{L}=C_{L} \frac{k}{\varepsilon}
$$

where $C_{L}$ is a constant to be determined and is not well known. By matching the diffusivity of tracer particles to the scalar diffusion rate predicted by the turbulence model, $v_{t} / \sigma$, the Lagrangian integral time scale becomes:

$$
T_{L} \approx 0.15 \frac{k}{\varepsilon}
$$

In the stochastic discrete random walk model, the interaction of a particle with a succession of discrete fluid phase turbulence eddies is simulated. Each eddy is characterized by a Gaussian distributed random velocity fluctuation, $u^{\prime}, v^{\prime}$, and a time scale $\tau_{e}$. By using the assumption of a Gaussian probability distribution,

$$
u^{\prime}=\zeta \sqrt{\overline{u^{\prime 2}}}
$$


where $\zeta$ is a normally distributed random number, the remainder of the right-hand side is the local root mean square (R.M.S) value of the velocity fluctuations. At each point in the flow, the value of the R.M.S fluctuating components at each point on the flow can be obtained from the kinetic energy as:

$$
\sqrt{\overline{u^{\prime 2}}}=\sqrt{\overline{v^{\prime 2}}}=\sqrt{2 k / 3}
$$

The lifetime of the eddy is defined as a random variation about $T_{L}$ :

$$
\tau_{e}=-T_{L} \log (r)
$$

where $r$ is a uniform random number between 0 and 1 . The particle is assumed to interact with the fluid phase eddy over this eddy lifetime. When the eddy lifetime is reached, a new value of the instantaneous velocity is obtained by applying a new value of $\zeta$ in Eq. (22).

In Eq. (10), the fluid is time-averaged and is considered to be constant during a particle time step. In order to improve the accuracy of pathline calculation and particle trajectory calculation, the fluid phase velocity at the $n+1$ time level is approximated by

$$
u^{n+1}=u^{n}+\Delta t \nabla u \cdot u_{p}^{n}
$$

The technique of prediction and correction of the fluid velocity has been detailed in previous researches [30]. It is therefore expected that higher accuracy of the continuous phase pathline and particle trajectory can be achieved.

\section{Results and discussions}

Numerical simulations are performed on a 2D axisymmetric domain. The spatial discretization of a variable employed the following schemes: second order upwind differencing scheme for $k-\varepsilon$, second order for pressure, QUICK (quadratic upwind interpolation of convection kinematics)[31] for momentum, PISO (Pressure-implicit 
with splitting of operators) [32] for pressure-velocity coupling. The Lagrangian tracking approach is adopted for particle tracking; particle phase and fluid phase interaction are taken into account by the coupled computation. The particle trajectory that is influenced by the turbulence is predicted by the discrete random walk tracking approach. Multi-injections are used for matching high mass flow rates. The results and discussions are shown in the following sections.

\subsection{Present simulation of cloud expansion}

Present simulations are shown in Fig.1. The morphology development of the powder cloud is well reproduced at each interval of time. The growth and shape of the cloud are very similar with the experimental images mentioned in the next section. Fig. 2 shows the simulation results plotted by the contour of DPM concentration, velocity magnitude, vorticity magnitude and stream function. These graphs show that the flow field of the cloud expansion is well predicted numerically and provide an insight into the developing cloud for further consideration of design optimisation.

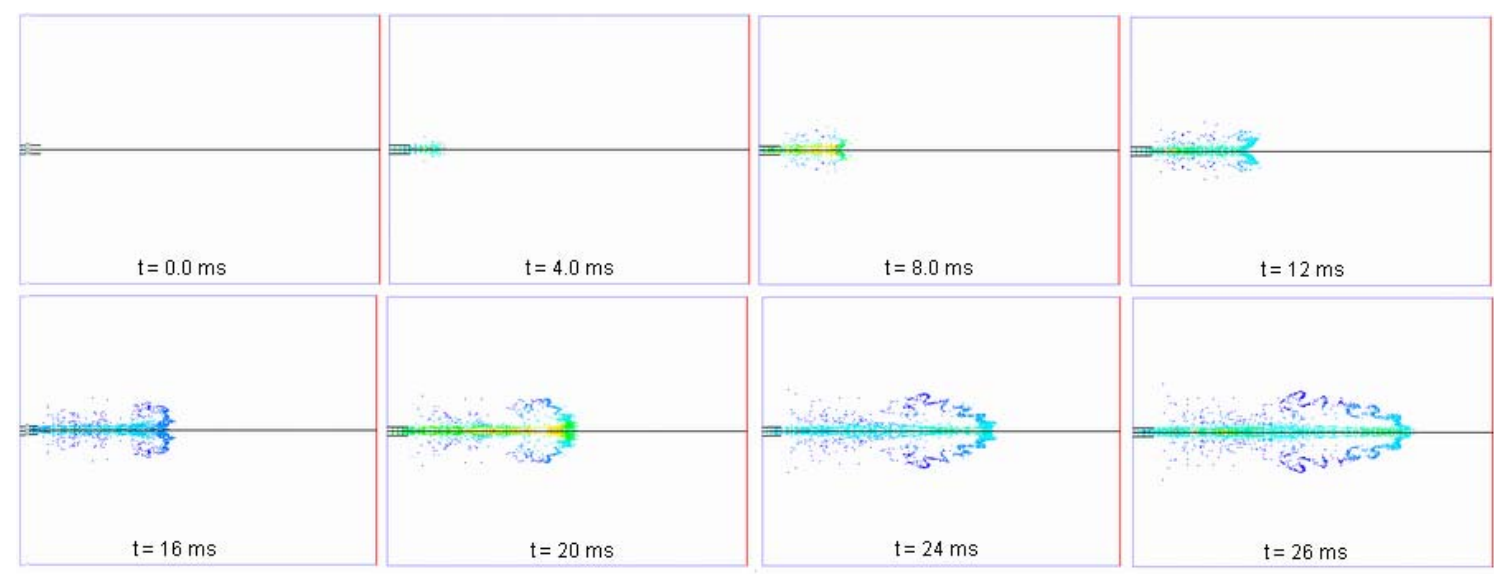

Fig.1 Morphology development of expansion cloud from a powder jet flow, growth of particle positions at time $\mathrm{t}=0,4,8,12,16,20,24,28 \mathrm{~ms}$. 


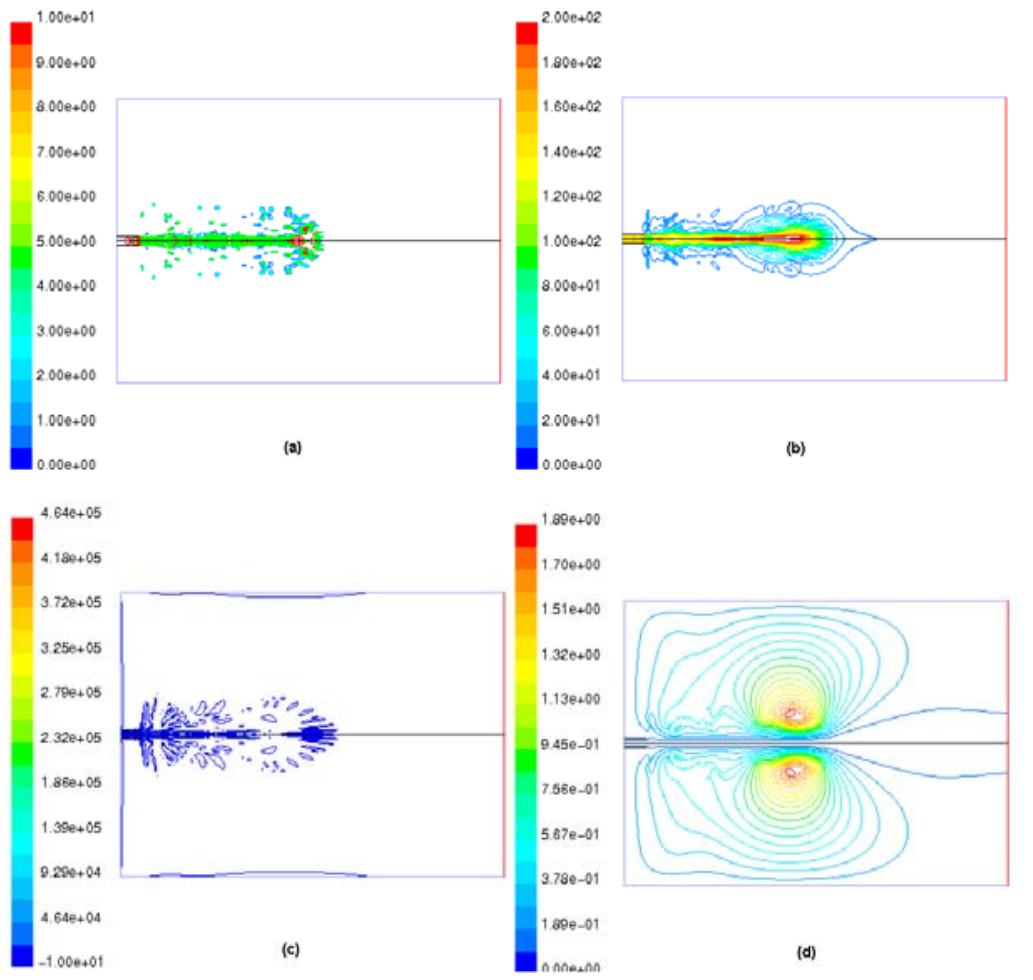

Fig.2 Simulation of expansion cloud: contour of (a) concentration, (b) velocity magnitude, (c) vorticity magnitude and (d) stream function at time $\mathrm{t}=20 \mathrm{~ms}$.

\subsection{Comparison with experimental images}

By comparing the morphological graphs of numerical simulations and experimental images [33] shown in Fig.3 (a) and (b), respectively, it is found that good agreement is obtained with the present simulation. The front shape of the cloud expansion is quite flat at the times 12 and $16 \mathrm{~ms}$, and these characteristics were similar to the experimental results. The development of the cloud expansion in this case is very important, since the behaviour of the expansion is the main concern for efficiency of suppression devices. The information from the numerical simulations will be useful to optimise the design of nozzles. 


\subsection{Comparison with previous simulation}

Previous simulation results [33] are shown in Fig.3 (c). Comparing with present simulations depicted in Fig.3 (a), improvements can be observed. The coverage of the expansion cloud including its developing length, width and shape are closer to the experimental results. This shows that the development of the expansion cloud has been reproduced numerically better than in the previous prediction. The reason is that the present study uses more accurate approaches such as particle interaction and particle-gas interaction algorithms, multi-particle injection methods, RNG $k-\varepsilon$ turbulence model, and more reasonable initial and boundary conditions that are mentioned in section 3 .

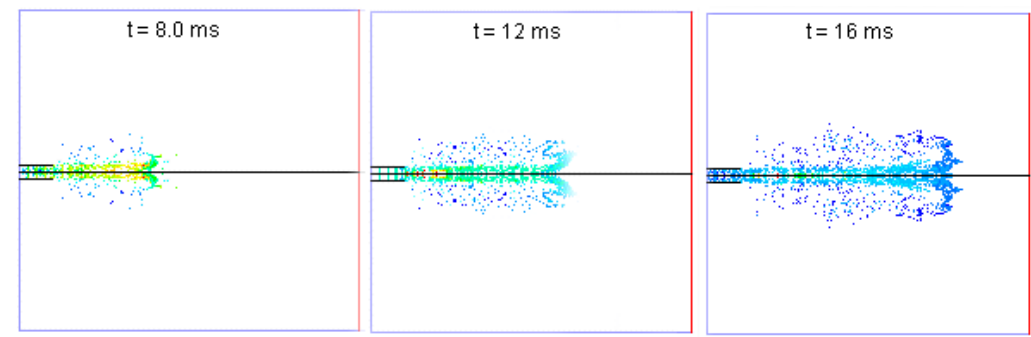

(a)

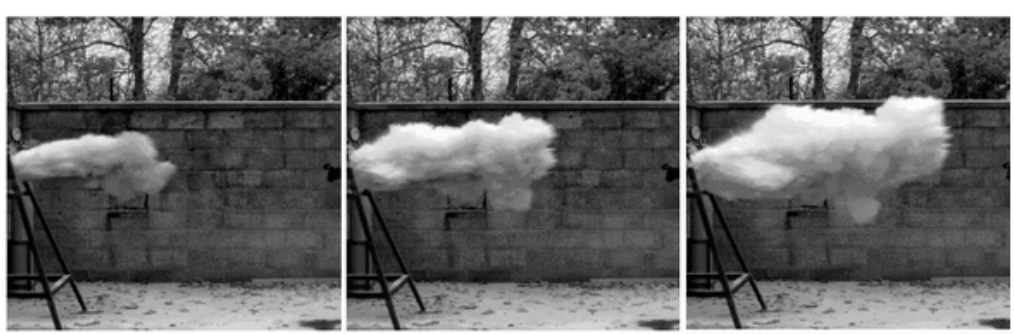

(b)

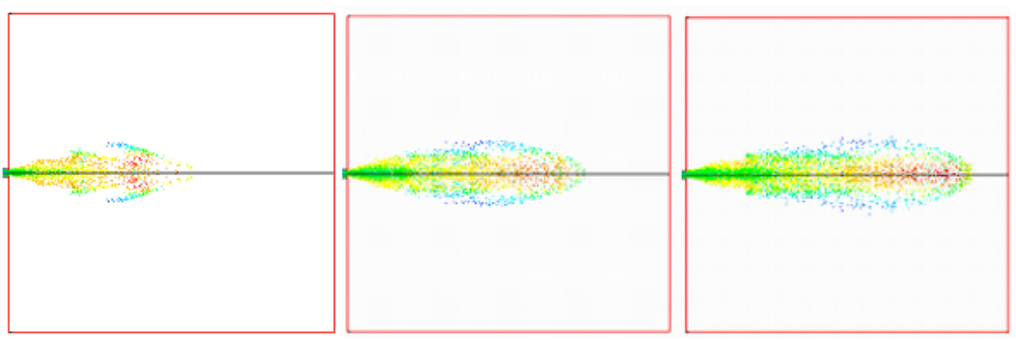

(c)

Fig.3 Comparison of development of morphology of expansion cloud and powder dispersion at time $\mathrm{t}=8,12,16 \mathrm{~ms}$, (a) present simulation; (b) experimental captured images [33] and (c) previous simulation [33]. 


\subsection{Comparison with experimental data}

Comparisons of numerical and experimental results [33] are shown in Fig.4, for the developing length of the expansion cloud and the effects of mass flow rate. Fig.5 shows the developing width of the expansion cloud and the effects of mass flow rate. The developing length of the expansion cloud is more influenced by the mass flow rates of the jet flow than the developing width. This means that the length of coverage of the powder jet flow can be easily controlled by the mass flow rates. However, the width of the coverage is only influenced by very high mass flow rates.

Comparisons of the velocity magnitude along the axis at time $\mathrm{t}=28 \mathrm{~ms}$ are shown in Fig.6, where V/V is the ratio of the local velocity/inlet velocity. Fig.7 shows the turbulent intensity along the axis at time $\mathrm{t}=28 \mathrm{~ms}$. The comparison shows that the present numerical investigations still produce results of qualitative level. Further improvements of the present numerical approach are needed for a detailed quantitative parametric study. These may include modified turbulent models that can be validated by the experiments. Fig. 8 shows the level of concentration of particles within the flow by modifying the constants of the $k-\varepsilon$ turbulence model. These results match more closely those of the experimental data. Further improvements should include a corrected particle tracking algorithm to improve the particle trajectories and increase capability of volume fraction, optimising the numerical procedure to achieve more stable and fast convergence, establishing a multi-physics reaction model to take into account possible gas - suppression powder reaction during development of the cloud, as well as a set of sophisticated experimental devices to obtain more accuracy in improving the physical models of powder jet flow and the initial and boundary conditions for the simulation. 


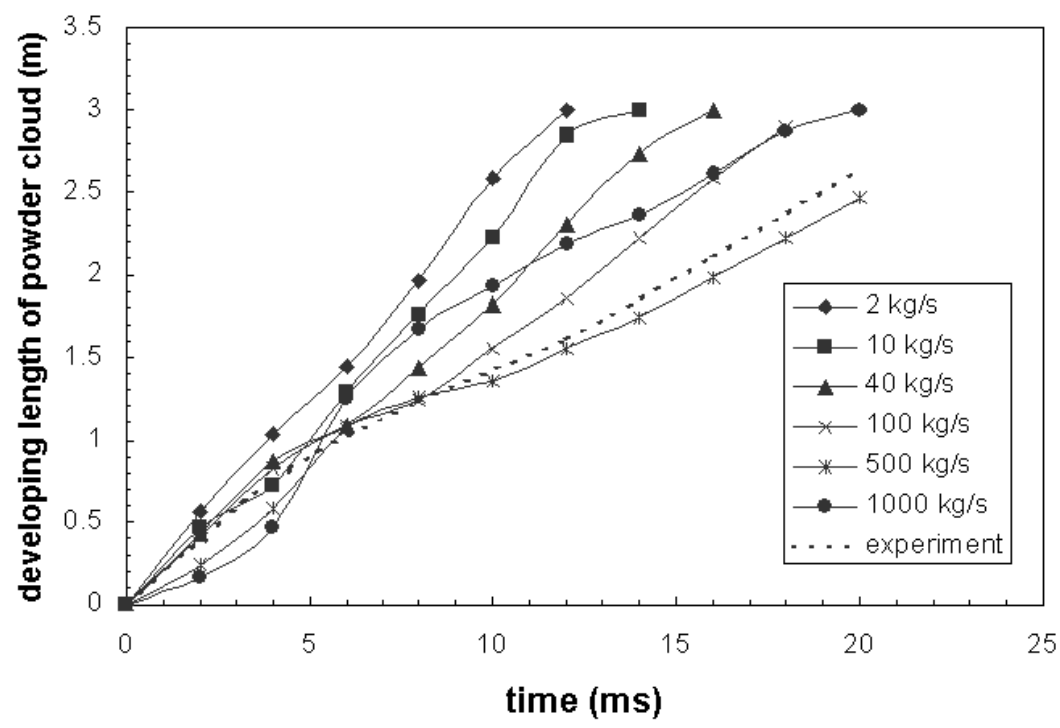

Fig.4 Comparison of numerical and experimental results for developing length of the expansion cloud and the effects of the mass flow rate

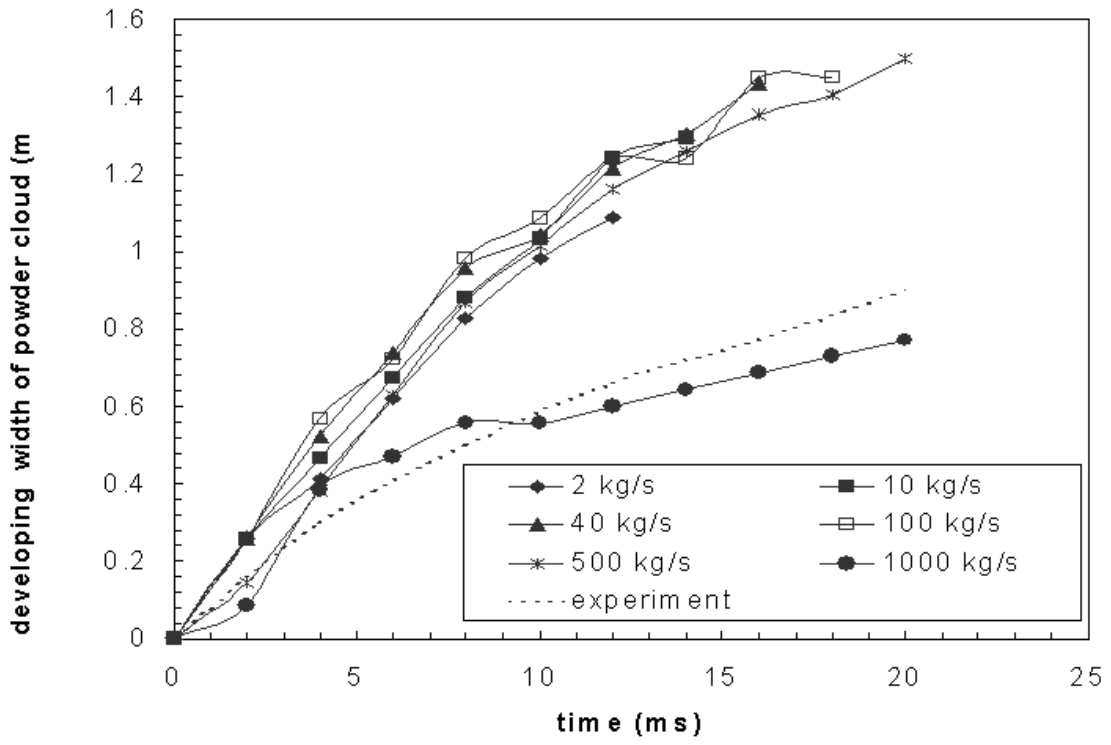

Fig.5 Comparison of numerical and experimental results for developing width of expansion cloud and the effects of the mass flow rate 


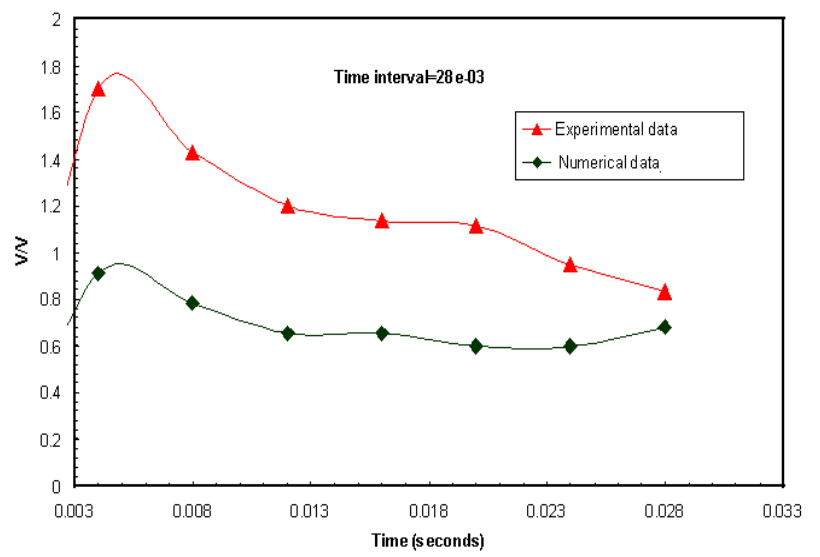

Fig. 6 comparison of velocity magnitude along the axis at time $\mathrm{t}=28 \mathrm{~ms}$.

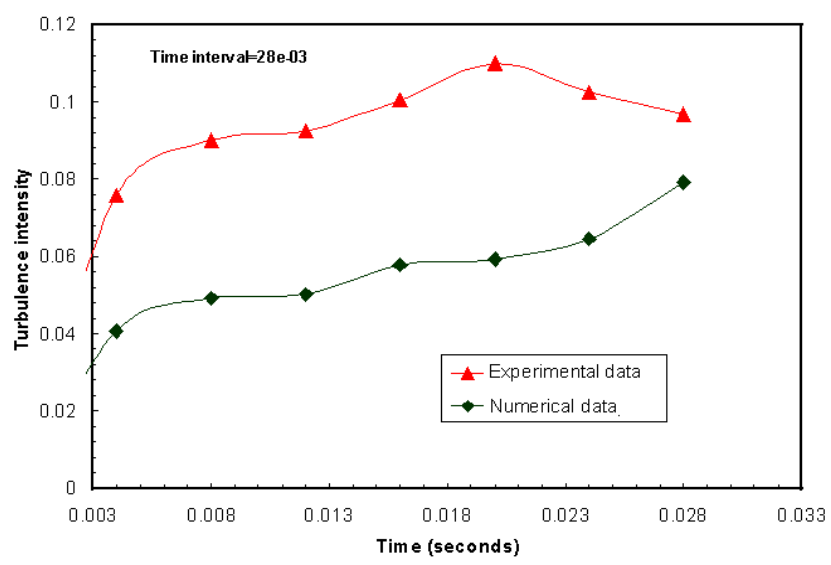

Fig. 7 comparison of turbulent intensity along the axis at time $\mathrm{t}=28 \mathrm{~ms}$

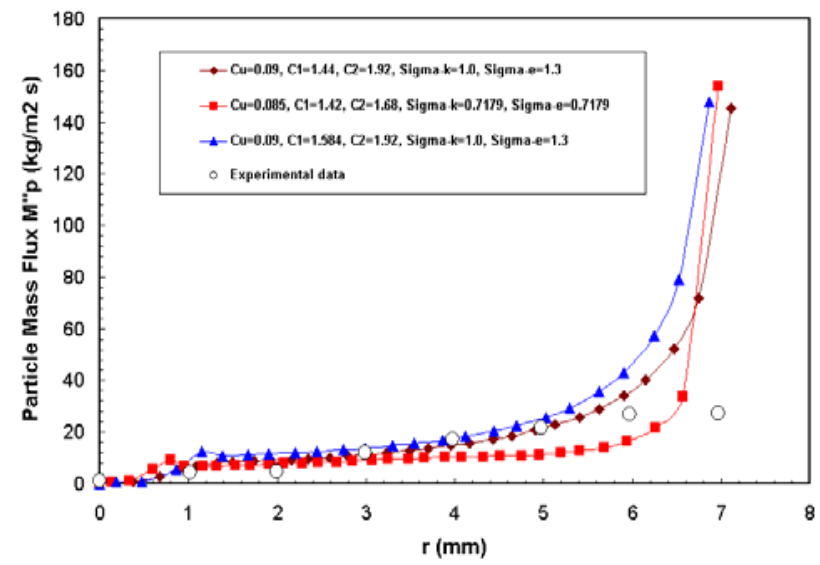

Fig.8 Comparison of modified turbulent models for particle concentrations 


\section{Conclusions}

This paper shows that numerical methods are capable of simulating the expansion cloud of a powder jet flow. The simulations employed the Lagrangian dispersed phase approach, the RNG $k-\varepsilon$ turbulence model and the particle-gas two-way momentum coupled algorithm in the flow field solver. The morphology of the developing cloud is demonstrated numerically. Qualitative agreements are achieved in comparison with experimental results. Though the present study is focused on a qualitative analysis, the simulation model can still be used to obtain an insight into the development process of the expansion cloud. This aims to provide a guide to the design of dispersion devices such as nozzles of extinguishing equipment in order to reduce trial and error experiments for optimising parameters. Further improvements and suggestions for increasing the accuracy of the numerical analysis have been briefly discussed.

\section{Acknowledgements}

The first three authors would like to acknowledge support from EPSRC, Kidde International Research, UK and the Mechanical Engineering Department at Brunel University. We are also grateful to researchers in CFD group and experimental group for helpful discussions on numerical approaches and the experimental process.

\section{References}

[1] Moore PE. Suppressants for the control of industrial explosions. J Loss Prev Process Ind 1996; 9(1):119-123.

[2] Eckhoff RK. Dust Explosions in the Process Industries. Oxford: Butterworth-Heinemann; 1991.

[3] Moore PE.. Propagation and Suppression of Gas and Dust Explosions. PhD Thesis, University of Surrey, Guildford, U.K. 1981.

[4] Hurlimann H. Explosionunterdruckung von staubexplosionen. VDI-Berichte 1989;701:617-657.

[5] Ogata K, Funatsu K, Tomita Y. Experimental investigation of a free falling powder jet and the air entranment. Powder Technology 2001; 115: 90-95

[6] Kennedy IM, Moddy MH. Particle dispersion in a turbulent round jet. Experimental Thermal and Fluid Science 1998; 18:11-26. 
[7] Anjorin VAO, Tang H, Morgan AJ, Barton IE. An experimental and numerical investigation into the dispersion of powder from a pipe. Experimental Thermal and Fluid Science 2003; 28: 45-54.

[8] Migdal D, Agosta DV. A source flow model for continuum gas-particle flow. Trans ASME J Appl Mech 1967; 34E 860.

[9] Sirignano, WA. Fluid dynamics of sprays - 1992 Freeman Scholar Lecture. J Fluid Eng 1993; 115: 345-378.

[10] Elghobashi S. On predicting particle-laden turbulent flows. Appl Sci Research 1994; 52: 309-324.

[11] Crow CT, Troutt TR, Chung JN. Numerical models for two-phase turbulent flows. Annual Rev Fluid Mech 1996; 28: 11-43.

[12] Taulbee DB, Mashayek F, Barre C. Simulation and Reynolds stress modeling of particleladen turbulent shear flows. Int J of Heat and Fluid Flow 1999;20: 368-373.

[13] Hagiwara Y., Takashins Y, Tanaka M. Direct numerical simulation of the basic phaseinteractions in liquid turbulence channel flow with immiscible droplets. Nuclear Engineering and Design 1997; 175: 49-57.

[14] Sato Y, Deutsch E, Simonin O. Direct numerical simulations of heat transfer by solid particles suspended in homogeneous isotropic turbulence. International Journal of Heat and Fluid Flow 1998; 19: 187-192.

[15] Chen X. Heavy particle dispersion in inhomogeneous, anisotropic, turbulent flows. Int J Multiphase Flow 2000; 26: 635-661.

[16] Singh P, Joseph DD, Hesla TI, Glowinski R, Pan TW. A distributed Lagrange multiplier/fictitious domain method for viscoelastic particulate flows. J of NonNewtonian Fluid Mechanics 2000; 91:167-188.

[17] Chen X., Pereira JCF. Computation of dispersed turbulent liquid-particle flows impinging a centerbody using an improved Lagrangian Stochastic model. Int Comm Heat Mass Transfer.1997; 24: 51-64.

[18] Launay K, Huilier D, Burnage H. Lagrangian predictions of the dispersion of heavy particles in a dilute two-phase flow: on the inertia effect. Mechanics Research Communications 1998; 25:251-256.

[19] Pokharna H, Mori M, Ransom VH. The particle fluid model and using Lagrangian representation in two-phase flow modelling. Nuclear Engineering and Design 1997; 175: 59-69.

[20] Pascal Ph, Oesterle B. On the dispersion of discrete particle moving in a turbulence shear flow. International Journal of Multiphase Flow 2000; 26: 293-325.

[21] Liu ZH, Zheng CG, Zhou LX. A second-order-moment-Monte-Carlo model for simulating swirling gas-particle flows. Powder Technology 2001; 120 (3):216-222.

[22] Barton IE. Simulation of particle trajectories in turbulent flow over a backward-facing step. R\&D Journal 1999; 15: 65-78.

[23] Morsi SA, Alexander AJ. An investigation of particle trajectories in two-phase flow systems. J Fluid Mech 1972; 55: 193-208.

[24] Choudhury D. Introduction to the Renormalization Group Method and Turbulence Modeling. Fluent Inc 1993; Technical Memorandum TM-107.

[25] Zhou Y, McComb WD, Vahala G. Renormalization Group (RG) in Turbulence: Historical and Comparative Perspective. NASA CR-201718 ICASE Report No 97-36, 
Institute for Computer Applications in Science and Engineering Mail Stop 403, NASA Langley Research Center Hampton, VA 23681-0001, August 1997, pp. 60.

[26] Kuo KKY. Principles of Combustion. Wiley Interscience, 1986, p.573-587.

[27] Bird GA. Molecular Gas Dynamics. Clarendon Press, Oxford, 1994.

[28] Gosman AD, Ioannides E. Aspects of computer simulation of liquid-fueled combustors. J Energy 1983; 7: 482-490.

[29] Tanaka T, Kiribayashi K, Tusji Y. Monte Carlo simulation of gas-solid flow in verticle pipe or channel. In: Proc Int Conf on Multiphase Flows, Tsukuba, Japan, 1991vol.2, p.439-442.

[30] Barton IE. Exponential-Lagrangian tracking schemes applied to Stokes law. Journal of Fluids Engineering, Transaction of the ASME, 1996; 118: 85-89.

[31] Leonard BP. A stable and accurate convective modelling procedure based on quadratic upstream interpolation. Compu Meth Appl Mech Eng 1979; 20: 23-25.

[32] Issa RI, Gosman AD, Watkins AP. The computation of compressible and incompressible recirculating flows by a non-iterative implicit scheme. J Comput Phys 1991; 93: 388410.

[33] Morgan AJ. The arresting of explosions to minimize environmental damage. $\mathrm{PhD}$ thesis, Brunel University, UK, 2000. 